\title{
Nearly logarithmic decay in the colloidal hard-sphere system
}

\author{
M. Sperl \\ Fachbereich Physik, Universität Konstanz, 78457 Konstanz, Germany
}

(Received 21 December 2004; revised manuscript received 25 February 2005; published 13 June 2005)

\begin{abstract}
Nearly logarithmic decay is identified in the data for the mean-squared displacement of the colloidal hardsphere system at the liquid-glass transition [W. van Megen et al., Phys. Rev. E 58, 6073 (1998)]. The solutions of the mode-coupling theory for the microscopic equations of motion fit the experimental data well. Based on these equations, the nearly logarithmic decay is explained as the equivalent of a $\beta$-peak phenomenon, a manifestation of the critical relaxation when the coupling between of the probe variable and the density fluctuations is strong. In an asymptotic expansion, a Cole-Cole formula including corrections is derived from the microscopic equations of motion, which describes the experimental data for three decades in time.
\end{abstract}

PACS number(s): 82.70.Dd, 64.70.Pf, 61.20.Lc

The transition of a fluid to a glass is accompanied by a dramatic slowing down of the dynamics as the system approaches the transition point [1]. The correlation function for some variable $A, \phi_{A}(t)=\left\langle A^{*}(t) A\right\rangle /\left\langle|A|^{2}\right\rangle$, with \langle\rangle denoting canonical averaging, decays rapidly, typically within a time increase by a factor of 10 in the normal fluid regime, while it stretches over orders of magnitude in times close to a glass transition. Simultaneously nontrivial dynamical features such as power-law decay and unconventional scaling are observed. The characterization and explanation of these signatures of the glass transition present challenges to experiment, computer simulation, and theory alike. One such feature is the nearly logarithmic decay of the correlation function, which was discovered recently in the orientational correlation function by optical-Kerr-effect (OKE) measurements in molecular liquids [2]. The response function measured in these experiments is proportional to the negative time derivative of the correlation function, so a measured nearly $t^{-1}$ decay is equivalent to a nearly logarithmic relaxation in $\phi_{A}(t)$. In a subsequent fit it was shown that the data can be well described by a schematic model within the modecoupling (MCT) theory for ideal glass transitions [3]. The nearly logarithmic decay was interpreted as a $\beta$-peak phenomenon of the short-time critical relaxation when the rotation-translation coupling is sufficiently large. Different from earlier suggestions, the presence of higher-order glass transitions, as in systems with very short-ranged attractions [4], could be ruled out as a cause for the nearly logarithmic decay in molecular systems. Similar OKE data were fitted by a kinetically constrained model [5].

Schematic models are truncated versions of the full MCT equations of motion that share the mathematical structure and universal properties of the latter but do not contain microscopic details [6]. Instead, a number of parameters in the schematic models need to be fitted. In the microscopic equations, these parameters are completely determined by the number density $\rho=N / V$ for a system of $N$ particles in a volume $V$, and the static structure factor $S_{q}$ given by the interaction potential [6-8]. In the following, a system of hard spheres of diameter $d$ shall be discussed, where the only control parameter is the packing fraction $\varphi=\pi \rho d^{3} / 6$. Predictions for the hard-sphere system (HSS) can be tested in colloidal suspensions, and the results for collective density fluctuations demonstrate the universal laws of MCT and verify certain characteristic parameters of the theory [9]. The tagged particle dynamics was analyzed recently in a computer-simulation study showing good agreement with MCT [10].

A particularly informative quantity to be studied in a system that shows structural relaxation is the mean-squared displacement (MSD), which is defined by $\delta r^{2}(t)$ $=\left\langle|\vec{r}(t)-\vec{r}(0)|^{2}\right\rangle$ with $\vec{r}(t)$ denoting the position of a particle at time $t$. The MSD is studied frequently in computer simulation and was measured for nine decades in time in a colloidal suspension [11]. In this paper, the MSD will be calculated for the HSS within MCT in order to show that for a state near the glass transition there is a window of two decades in time of nearly logarithmic relaxation. This will be done by identifying a $t^{-1}$ decay of the derivative of the MSD in complete analogy to the procedure used for the analysis of the corresponding result for the OKE data [2]. Second, by asymptotic solution of the MCT equations, a modified Cole-Cole law will be derived for the description of the critical decay of the MSD. This analytical formula explains the nearly logarithmic regime and accounts for the critical decay for three decades in time. Third, it will be shown that the MCT solutions at the critical point describe the experimental result in [11] for five orders of magnitude in time. Fourth, the full range of available experimental data for the MSD is described reasonably by the numerical solutions of the MCT equations going beyond the universal laws. Thus nearly logarithmic decay laws known from molecular systems as $\beta$-peak phenomenon are established in a colloidal system.

Slow dynamics within MCT originates from a singularity in the long-time behavior of the collective density fluctuations [8]. Other variables share that behavior if they are coupled to the density fluctuations. While the coupling to rotational motion was modeled by an empirical parameter in [3], the MSD for a colloidal system is given by a microscopic equation of motion by [12],

$$
\delta r^{2}(t)+D_{0}^{s} \int_{0}^{t} d t^{\prime} m^{(0)}\left(t-t^{\prime}\right) \delta r^{2}\left(t^{\prime}\right)=6 D_{0}^{s} t
$$

with the short-time diffusion coefficient $D_{0}^{s}$. The memory kernel $m^{(0)}(t)$ is determined by the static structure of the liquid and collective and single density correlation functions. 


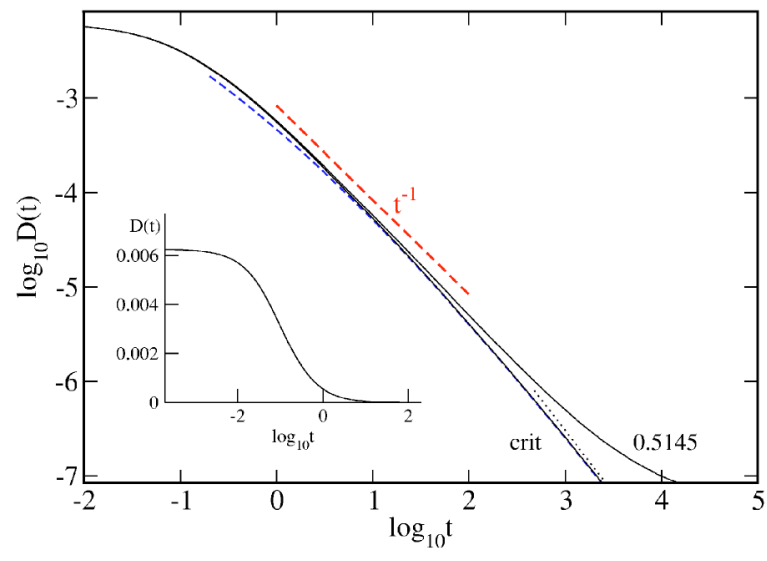

FIG. 1. (Color online.) Time derivative of the mean-squared displacement $D(t)$ in the hard-sphere system for packing fractions $\varphi=\varphi^{c}$ and $\varphi=0.5145$ (full lines from left to right). The upper dashed line is proportional to $t^{-1}$. The lower dashed curve represents the approximation by Eq. (4). The dotted line displays the critical law $t^{-a-1}$. Here and in Fig. 2 the unit of time is fixed by the short-time diffusion coefficient $D_{0}^{s} / d^{2}=1 / 160$. The inset shows $D(t)$ for $\varphi=\varphi^{c}$ on a linear scale for comparison.

The structure factor is calculated using the Percus-Yevick approximation (PYA) [7]; details of the numerical solution are outlined elsewhere [12]. For $\varphi$ below the critical point $\varphi^{c}=0.5159$, the long-time solution of Eq. (1a) is diffusive, $\delta r^{2}(t)=6 D^{s} t$, with a long-time diffusion coefficient $D^{s}$ depending on the distance from the critical point. For $\varphi \geqslant \varphi^{c}$, the long-time solution is arrested at a plateau $\delta r^{2}(t)=6 r_{s}^{2}$, with a localization length $r_{s}$. The derivative of the MSD can be interpreted as a time-dependent diffusion coefficient, $6 D(t)=d\left[\delta r^{2}(t) / d^{2}\right] / d t$, that goes to zero as the glass transition is approached [13]. $D(t)$ calculated from Eq. (1a) is plotted for $\varphi=0.5145$ and $\varphi=\varphi^{c}$ as full lines in Fig. 1. The dynamics when approaching the critical point shares more and more of the relaxation at $\varphi^{c}$ as seen for $\varphi=0.5145$ for $\log _{10} t<1$. This portion of the dynamics is therefore referred to as critical relaxation. For times exceeding the critical relaxation, the dynamics crosses over to the long-time diffusion as seen for $\varphi=0.5145$ around $\log _{10} t \approx 2$. The most significant finding in connection with Fig. 1 is the appearance of a window in time after the transient where the dynamics follows closely a $t^{-1}$ law for $0 \leqslant \log _{10} t \leqslant 2$. A comparison of the solution for $\varphi=0.5145$ with the critical decay shows that the major fraction of the $t^{-1}$ decay is part of the critical relaxation. This decay reflects the same behavior as found in molecular liquids, moreover, the decay observed in Fig. 1 is closer to $t^{-1}$ than for most of the OKE data where $t^{-x}$ is seen with $x$ ranging from 0.8 to 1.15 [2,3]. The long-time decay at the critical point is a $t^{-a}$ law with $a=0.312$ for the HSS. This law is shown as the dotted straight line of slope $-(1+a)$. It accounts for the critical decay only for $\log _{10} t \geqslant 3$, is preceeded by the $t^{-1}$ decay, and has no relevance for $\varphi$ $=0.5145$.

The nearly logarithmic decay is found adjacent to the transient for the Brownian colloidal system as for the Newtonian molecular systems [3]. For the HSS also, Newtonian dynamics can be considered and the equivalent of Eq. (1a) reads [14]

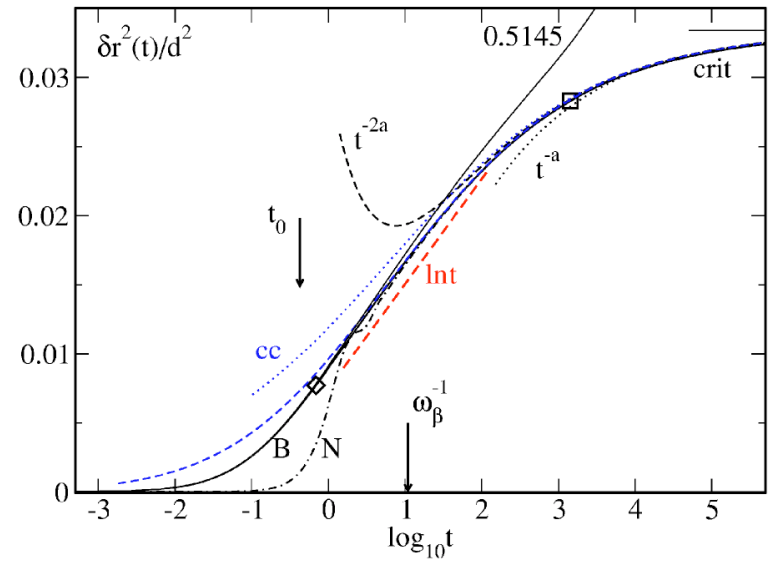

FIG. 2. (Color online.) Mean-squared displacement at the critical point in the hard-sphere system for Newtonian ( $N$, chain curve) and Brownian ( $B$, full curve) dynamics. The short horizontal line displays the plateau $6 r_{s}^{c 2}$. The dashed lines show the asymptotic approximations by Eqs. (2) (labeled $t^{-2 a}$ ) and (4). The leading-order approximations are shown dotted for Eq. (2) $\left(t^{-a}\right)$ and Eq. (4) $(c c)$. The nearly logarithmic relaxation is shown by the straight dashed line labeled $\ln t$. The diamond and the square indicate $10 \%$ deviations of $B$ from the approximation by Eq. (4) and the solution for $\varphi=0.5145$, respectively.

$$
\partial_{t} \delta r^{2}(t)+v_{s}^{2} \int_{0}^{t} d t^{\prime} m^{(0)}\left(t-t^{\prime}\right) \delta r^{2}\left(t^{\prime}\right)=6 v_{s}^{2} t
$$

with the thermal velocity $v_{s}^{2}$ for a tagged particle. Figure 2 shows the solution for the MSD for Newtonian and Brownian dynamics for $\varphi=\varphi^{c}$. Both solutions can be matched at long times and they overlap down to $\delta r^{2}(t) \approx 0.01$ defining the beginning of the regime for structural relaxation at $\log _{10} t \approx 0$ [14]. The nearly logarithmic decay is shown as the nearly linear increase of the MSD with $\log t$ outside the transient within the same time interval as marked by the dashed line in Fig. 1.

To further analyze the window of nearly logarithmic relaxation and in an attempt to distinguish it from a further approximation to a power law with a very small exponent as in [15], asymptotic expansions shall be applied. At the critical point, the MSD can be expanded in power laws and reads [12]

$$
\delta r^{2}(t) / 6=r_{s}^{c 2}-h_{M S D}\left(t / t_{0}\right)^{-a}+h_{M S D} K_{M S D}\left(t / t_{0}\right)^{-2 a},
$$

where the first two terms on the right-hand side constitute the leading-order result. The values for the HSS are $r_{s}^{c}=0.0746$, $h_{M S D}=0.0116, K_{M S D}=-1.23, t_{0}=0.425, \lambda=0.735$. The leading and next-to-leading order results of Eq. (2) are shown as dotted and dashed lines in Fig. 2 and describe the full solutions only for $t \gtrsim 1000$ and $t \gtrsim 100$, respectively. As will be shown below, the leading order fails to apply in the experimentally relevant window, while including the correction still does not explain more than one decade of structural relaxation.

In the following, an asymptotic solution is derived that is based on the expansion of the memory kernel. For long times, the equations of motion for the MSD are the same for 
both Newtonian and Brownian dynamics [6,14], and can be represented with the modified Laplace transform $\mathcal{S}[f(t)](z)$ $=i z \int_{0}^{\infty} d t \exp [i z t] f(t)$ as

$$
\mathcal{S}\left[\delta r^{2}(t)\right](z)=\frac{6}{\mathcal{S}\left[m^{(0)}(t)\right](z)} .
$$

An asymptotic expansion for the memory kernel equivalent to the one in Eq. (2) is given by $m^{(0)}(t)=f_{m}^{c}{ }_{m}^{(0)}+h_{m^{(0)}}\left(t / t_{0}\right)^{-a}$ $+h_{m}(0) K_{m}(0)\left(t / t_{0}\right)^{-2 a}$, with $f_{m}^{c}(0)=1 / r_{s}^{c 2}, h_{m}(0)=h_{M S D} / r_{s}^{c 4}, K_{m(0)}$ $=K_{M S D}+\lambda h_{M S D} / r_{s}^{c 2}$, and $\lambda=\Gamma(1-a)^{2} / \Gamma(1-2 a)$ with the Euler gamma function $\Gamma(x)$. In the Laplace domain, this can be written with a characteristic frequency $\omega_{\beta}$ as $\mathcal{S}\left[m^{(0)}(t)\right](z)=f_{m}^{c(0)}\left\{1+\left(-i z / \omega_{\beta}\right)^{a}+K_{M S D}^{c c}\left(-i z / \omega_{\beta}\right)^{2 a}\right\}$, where $K_{M S D}^{c c}=f_{m(0)}^{c} K_{m}(0) /\left(h_{m(0)} \lambda\right)$. Hence, Eq. (3) reads up to next-toleading order

$$
\mathcal{S}\left[\delta r^{2}(t)\right](z)=\frac{6 r_{s}^{c 2}}{1+\left(-i z / \omega_{\beta}\right)^{a}+K_{M S D}^{c c}\left(-i z / \omega_{\beta}\right)^{2 a}},
$$

and the characteristic frequency is given by

$$
\omega_{\beta}=\frac{1}{t_{0}}\left[\frac{r_{s}^{c 2}}{h_{M S D}} \frac{1}{\Gamma(1-a)}\right]^{1 / a} .
$$

For the HSS we get $t_{0} \omega_{\beta}=0.03895$. The leading-order result in Eq. (4) is obtained for $K_{M S D}^{c c}=0$, and is known as ColeCole law. For different variables a similar result was obtained before $[3,16]$. While the correction in Eq. (2) is comparably large, $K_{M S D}=-1.23$, the analogous result for the memory kernel is only a fraction of it, $K_{m}(0)=0.30$. The correction in Eq. (4) is even smaller, $K_{M S D}^{c c}=0.196$. As the corrections determine the range of validity for the leading terms of the asymptotic expansions, the leading-order result of Eq. (4) is superior to the leading-order result of Eq. (2) as seen in Fig. 2, where already the leading order of Eq. (4) describes qualitatively the complete relaxation. Including the correction explains the complete window of structural relaxation as seen by the diamond marking a $10 \%$ deviation of the approximation by Eq. (4) from the solution of Eq. (1a). A comparison in Fig. 1 shows that Eq. (4) also covers the complete regime of nearly logarithmic decay. For $\varphi=0.5145, D(t)$ in Fig. 1 and the MSD in Fig. 2 is described by Eq. (4) for $\log _{10} t \leqslant 3$. The crossover from the critical decay to the longtime diffusion yields an extended window in time where $\ln t$ fits the solution.

Figure 3 shows the data from [11] in units of the particle radius $R=d / 2$ and the Brownian time scale $\tau_{b}=R^{2} /\left(6 D_{0}^{s}\right)$ together with the solutions of Eq. (1a) for different values of $\varphi$. The agreement is rather satisfactory considering that all coupling parameters are fixed microscopically. Three adjustments need to be made to match experimental and theoretical curves. First, the short-time diffusion coefficient sets an overall time scale that needs to be accounted for. The theoretical solutions are matched to the data for $\log _{10} t \leqslant-1$. As the experimental curves do not fall on top of each other in that regime, the chosen time scale also needs to vary by a factor up to 2 . Second, with $\varphi^{c} \approx 0.58[11,17]$, the experimental glass transition in the HSS is found at approximately

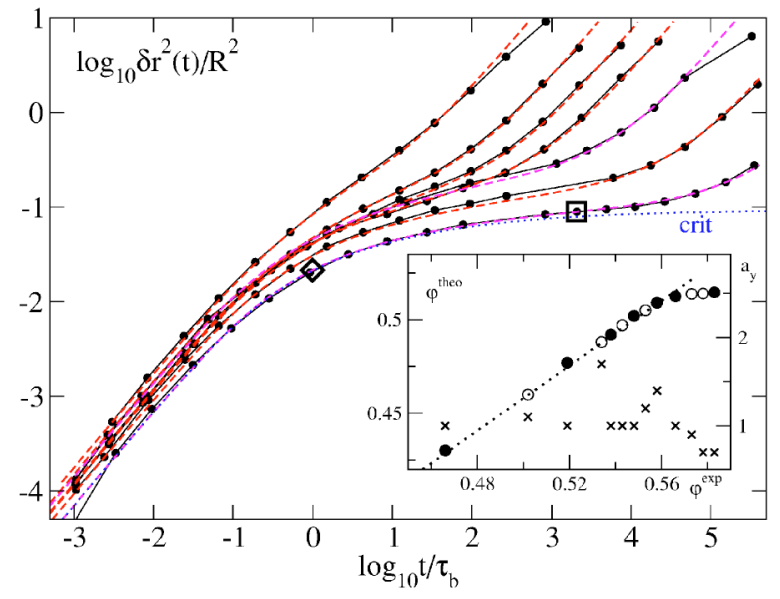

FIG. 3. (Color online.) Fit of the mean-squared-displacement data from [11] (full circles and curves) by the solutions of modecoupling theory (dashed). The dotted curve displays the theoretical result at the critical point and is equivalent to curve $B$ in Fig. 2. The square indicates a $10 \%$ deviation between the critical relaxation and the fit for $\varphi^{\exp }=0.583$. The open diamond is the same as in Fig. 2. The inset shows the mapping of the experimental packing fractions $\varphi^{\exp }$ onto theoretical values $\varphi^{\text {theo }}$ on the left axis as closed and open circles for curves shown or left out, respectively. The dotted line shows the function $\varphi^{\text {theo }}=0.87 \varphi^{\exp }+0.023$. The right axis displays the vertical scaling factor by crosses.

a $12 \%$ higher packing fraction than predicted in Refs. $[8,12]$. This discrepancy could be reduced to below $10 \%$ by using the actual structure factor rather than the PYA in a computer simulation [18], but a remaining error from the modecoupling approximation has to be accepted. This is similar to other approaches fitting the data [19]. Therefore, theoretical values for $\varphi$ are mapped on experimental ones by comparing the diffusivity in experiment and theory. The result of this procedure is shown in the inset of Fig. 3 by circles. The mapping is fitted by $\varphi^{\text {theo }}=0.87 \varphi^{\text {exp }}+0.023$, which is remarkably similar to what was found from a computer simulation of the HSS [10], and extrapolates to a critical density $\varphi^{c}$ $\approx 0.57$. The highest values of $\varphi$ do not follow that linear mapping. This is due to aging for $\log _{10} t / \tau_{b} \geqslant 4$, where the experiment is no longer fully equilibrated $[11,17]$. The dynamics before aging sets in, and hence the regime for nearly logarithmic relaxation, is not affected significantly by aging [17]. Third, as the actual transition takes place at a higher packing fraction than theory predicts, a smaller localization length and hence a vertical shift of the curves by a prefactor $a_{y}$ - shown by crosses in the inset of Fig. 3-can be expected. Surprisingly, many curves are described well using $a_{y}=1$. The variation of $a_{y}$ yields an error estimate for the localization length of around $20 \%$ for the localization length as compared to the theoretical results. If only data close to the glass transition is considered, the data show indeed a smaller localization length than predicted. After these adjustments, Eq. (1a) fits the data for different packing fractions over the entire experimental range of up to nine orders of magnitude in time.

The curve $\varphi^{\exp }=0.583$ is fitted by $\varphi^{\text {theo }}=0.5146$, and in addition, the critical relaxation for $\varphi^{\text {theo }}=\varphi^{c}$ is matched with the same prefactors and shown dotted. The data follow 
closely the critical relaxation for $t / \tau_{b} \lesssim 2000$. The two theoretical curves correspond to the ones shown in Figs. 1 and 2, thus establishing the existence of a window of nearly logarithmic relaxation in the experimental data.

In conclusion, it is shown in Fig. 3 that the mode-coupling theory is able to describe the mean-squared displacement in a colloidal hard-sphere system [11] rather accurately. This implies consistency with both universal laws of MCT and nonuniversal MCT predictions for the HSS. Outside the transient, the data exhibit a window of two orders of magnitude in time where the relaxation resembles nearly logarithmic decay or a $\beta$-peak phenomenon (cf. Fig. 1). The latter is found independent of the underlying Brownian dynamics also for Newtonian dynamics (cf. Fig. 2), and is distinguished from an accidental crossover by an asymptotic expansion relating it to a $\beta$ peak (cf. Eq. (4)). Such a dynamical feature was discovered in molecular systems [2] and interpreted as a consequence of strong translation-rotation coupling [3]. In addition, similar nearly logarithmic decays were also observed in nematic liquid crystals [20]. For the hardsphere system, while clearly lacking rotational degrees of freedom, the MSD constitutes another variable that is coupled strongly to the density fluctuations if the tagged particle is sufficiently large. It can be inferred from Ref. [12] that $m^{(0)}(t)$ in Eq. (1) becomes smaller when the size of the tagged particle is chosen smaller than the particles in the host fluid. A larger particle in turn experiences a stronger coupling of its MSD to the collective dynamics. As for increasing rotation-translation coupling in [3], increasing the diameter of the particle for which the MSD is measured enhances the deviation between the underlying collective dynamics and the coupled variable. The empirical nearly logarithmic law is part of the critical relaxation and does not change slope when observed sufficiently close to the transition point. This is in clear contrast to the logarithmic laws in the vicinity of higher-order glass-transition singularities [4] and can be used to distinguish both relaxation features. Hydrodynamic interactions present in colloidal suspensions are not included in Eq. (1) but are known to change the short-time dynamics in MCT [21]. The quality of the data fit and the validity of the nearly logarithmic law are therefore surprising and rule out a significant contribution of hydrodynamic interactions on the dynamics of the MSD within MCT as shown in Fig. 3. This is in contrast to other approaches $[15,19]$. This can be rationalized by the strong coupling of the MSD to the collective dynamics causing the MSD for a larger particle to be slower than for a smaller particle [12]. For the slowest collective relaxations only small deviations by hydrodynamic interactions have been found [21], a result that apparently also applies to the slow coupled variable MSD.

Equation (4) improves the understanding of glassy dynamics in three aspects. First, it comprises a Cole-Cole that is derived rigorously from microscopic equations of motion and therefore provides a foundation for data interpretations as in Ref. [3]. Second, it closes a gap in the understanding of the MSD close to the glass transition [14]. Together with the results from Ref. [12], MCT provides analytical descriptions of the dynamics in all subsequent windows in time up to small crossover regions: The initial ballistic or Brownian dynamics is followed by a critical relaxation described by Eq. (4), before a plateau $6 r_{s}^{c 2}$ is reached. After that plateau, the crossover to the long-time diffusion starts with a von Schweidler law $t^{-b}$ where $b=0.583$ [12]. Third, precise meaning is given to the finding that strongly coupled variables do not necessarily show the same dynamics.

This work was stimulated by discussions with H.Z. Cummins, M. Fuchs and W. Götze, and supported by the DFG SFB 513 and the DFG Grant No. SP 714/3-1.
[1] W. Kob, in Slow Relaxations and Nonequilibrium Dynamics in Condensed Matter, edited by J.-L. Barrat, M. Feigelman, J. Kurchan, and J. Dalibard (Springer, Berlin, 2003), p. 199.

[2] G. Hinze, D. D. Brace, S. D. Gottke, and M. D. Fayer, Phys. Rev. Lett. 84, 2437 (2000); 84, 4783(E) (2000); J. Chem. Phys. 113, 3723 (2000); M. Ricci, P. Bartolini, and R. Torre, Philos. Mag. B 82, 541 (2002); H. Cang, V. N. Novikov, and M. D. Fayer, Phys. Rev. Lett. 90, 197401 (2003); J. Chem. Phys. 118, 2800 (2003).

[3] W. Götze and M. Sperl, Phys. Rev. Lett. 92, 105701 (2004).

[4] M. Sperl, Phys. Rev. E 68, 031405 (2003); F. Sciortino, P. Tartaglia, and E. Zaccarelli, Phys. Rev. Lett. 91, 268301 (2003).

[5] L. Berthier and J. P. Garrahan, J. Phys. Chem. B 109, 3578 (2005).

[6] W. Götze, in Liquids, Freezing and Glass Transition, edited by J. P. Hansen, D. Levesque, and J. Zinn-Justin (North Holland, Amsterdam, 1991), p. 287.

[7] J.-P. Hansen and I. R. McDonald, Theory of Simple Liquids, 2nd ed. (Academic, London, 1986).

[8] U. Bengtzelius, W. Götze, and A. Sjölander, J. Phys. C 17, 5915 (1984).

[9] W. van Megen, Transp. Theory Stat. Phys. 24, 1017 (1995).
[10] T. Voigtmann, A. Puertas, and M. Fuchs, Phys. Rev. E 70, 061506 (2004).

[11] W. van Megen, T. C. Mortensen, S. R. Williams, and J. Müller, Phys. Rev. E 58, 6073 (1998).

[12] M. Fuchs, W. Götze, and M. R. Mayr, Phys. Rev. E 58, 3384 (1998).

[13] P. N. Segrè and P. N. Pusey, Phys. Rev. Lett. 77, 771 (1996).

[14] S.-H. Chong, W. Götze, and M. R. Mayr, Phys. Rev. E 64, 011503 (2001).

[15] M. Tokuyama, Y. Terada, and I. Oppenheim, Physica A 307, 27 (2002).

[16] W. Götze and L. Sjögren, J. Phys.: Condens. Matter 1, 4183 (1989).

[17] N. B. Simeonova and W. K. Kegel, Phys. Rev. Lett. 93, 035701 (2004).

[18] G. Foffi, W. Götze, F. Sciortino, P. Tartaglia, and T. Voigtmann, Phys. Rev. E 69, 011505 (2004).

[19] M. Tokuyama, H. Yamazaki, and Y. Terada, Phys. Rev. E 67, 062403 (2003); M. Tokuyama, Physica A 289, 57 (2001).

[20] H. Cang, J. Li, V. N. Novikov, and M. D. Fayer, J. Chem. Phys. 119, 10421 (2003).

[21] M. Fuchs and M. R. Mayr, Phys. Rev. E 60, 5742 (1999). 